\title{
Nonclassical correlation properties of radiation fields
}

\author{
Werner Voge* \\ Arbeitsgruppe Quantenoptik, Institut für Physik, Universität Rostock, D-18051 Rostock, Germany
}

\begin{abstract}
A full characterization of nonclassical space-time dependent correlations of radiation is formulated in terms of normally and time-ordered field correlation functions. It describes not only the properties of initially prepared multimode radiation fields, but also the dynamics of radiation sources. Some of these correlation effects occur in the resonance fluorescence of a single two-level atom.
\end{abstract}

PACS numbers: 03.70.+k, 42.50.Dv, 42.50.Ar

Nonclassical effects of radiation have not lost any part of their attraction since the early days of quantum physics [1, 2, 3]. It took over seven decades until Einsteins postulate of the existence of photons could be verified by a clear demonstration of the antibunching of photons [4]. Other demonstrations of nonclassical radiation properties, such as sub-Poissonian photon statistics [5] and quadrature squeezing [6] were following soon.

In the field of quantum optics the study of nonclassical effects of radiation was widely based on the GlauberSudarshan $P$-function [7, 8]. Whenever it fails to have the properties of a probability density, then the state is said to be nonclassical [9, 10]. This condition describes prominent examples of nonclassical effects.

Recently some possibilities of a complete characterization of nonclassicality have been developed for a single mode of the radiation field. Observable conditions could be derived, which are based on characteristic functions [11, 12] or on moments [13, 14]. Both approaches lead to infinite hierarchies of nonclassicality conditions in terms of the corresponding quantities. The characteristic function approach has already been used in some experiments [15, 16].

Nonclassicality of multimode radiation has been considered in some special cases only. Entanglement, a special nonclassical property, is considered to be useful for quantum information processing. A complete characterization in terms of moments is known for bipartite continuous-variable entangled states whose partially transposed density operator exhibits some negativities [17], for some special cases see also [18, 19]. There exist attempts to generalize the method for the multimode case [20] and for bound entanglement [21].

In the present Letter we provide a general characterization of nonclassical correlation properties of radiation fields in terms of space-time dependent (normally and time-ordered) field correlation functions. Beyond the characterization of initially prepared quantum states of free multimode radiation, this approach also describes the nonclassical effects caused by the dynamics of radiation sources. The physical realization and the detection of the new correlation effects are also considered.

Let us deal with field correlation properties in an arbitrary but fixed number of $k$ space-time points. In prac- tice this number will depend on the used detection setup. Consider an operator function $\hat{f}$,

$$
\hat{f} \equiv \hat{f}\left[\hat{E}^{(-)}(1), \ldots, \hat{E}^{(-)}(k), \hat{E}^{(+)}(k), \ldots, \hat{E}^{(+)}(1)\right],
$$

depending on the positive and negative frequency parts of the electric field operators, $\hat{E}^{( \pm)}(i)$, where $i \equiv\left(\mathbf{r}_{i}, t_{i}\right)$ is the space-time argument $(i=1, \ldots, k)$. We expand the operator $\hat{f}$ as

$$
\begin{array}{r}
\hat{f}=\sum_{\left\{n_{i}, m_{i}\right\}=0}^{\infty} c_{\left\{n_{i}, m_{i}\right\}}\left[\hat{E}^{(-)}(1)\right]^{n_{1}} \ldots\left[\hat{E}^{(-)}(k)\right]^{n_{k}} \\
\times\left[\hat{E}^{(+)}(k)\right]^{m_{k}} \ldots\left[\hat{E}^{(+)}(1)\right]^{m_{1}},
\end{array}
$$

where the notation $\left\{n_{i}, m_{i}\right\}$ is used for the dependence on $n_{1}, \ldots, n_{k}, m_{1}, \ldots, m_{k}$.

Classicality of normally and time-ordered correlation properties can be defined as

$$
\forall \hat{f}: \quad\left\langle\circ{ }_{\circ} \hat{f}^{\dagger} \hat{f}_{\circ}^{\circ}\right\rangle \geq 0,
$$

which generalizes the single-mode condition [14]. The $\therefore \ldots \circ$ notation (cf. [22]) denotes both normal (: $\cdots$ : notation) and time-ordering, with increasing times in the fields $\hat{E}^{(-)}$and $\hat{E}^{(+)}$from left to right and right to left, respectively. By generalizing the standard definition of the $P$-function (see $7,8,22]$ ) to the functional

$$
P\left[\left\{E^{(+)}(i)\right\}\right]=\left\langle\circ \prod_{i=1}^{k} \hat{\delta}\left(\hat{E}^{(+)}(i)-E^{(+)}(i)\right) \stackrel{\circ}{ }\right\rangle,
$$

the field correlation functions can be formally written as those of classical stochastic processes. They behave like classical ones, if $P\left[\left\{E^{(+)}(i)\right\}\right]$ has the properties of a classical joint probability density. In the classical limit the operators correspond to classical quantities: $\hat{E}^{( \pm)}(i) \rightarrow E^{( \pm)}(i)$ and $\hat{f} \rightarrow f$, ordering prescriptions are no longer needed, and averages become classical ones, $\langle\ldots\rangle_{\mathrm{cl}}$. In this case the left-hand side (lhs) of Eq. (3),

$$
\left\langle\circ \hat{f}^{\dagger} \hat{f}_{\circ}^{\circ}\right\rangle \rightarrow\left\langle|f|^{2}\right\rangle_{\mathrm{cl}}
$$

is indeed nonnegative in general.

Based on this results, a radiation field shows nonclassical normally and time-ordered correlation properties in $k$ space-time points, iff

$$
\exists \hat{f}: \quad\left\langle\circ \hat{f}^{\dagger} \hat{f}_{\circ}^{\circ}\right\rangle<0 .
$$


This completely defines the nonclassical correlation effects in the chosen space-time points. It is difficult, however, to handle the nonclassicality condition in this form. The operator $\hat{f}$ must be considered for all choices of the coefficients $c_{\left\{n_{i}, m_{j}\right\}}$. Moreover, it is unclear how to ob- serve the quantity $\left\langle\stackrel{\circ}{\circ} \hat{f}^{\dagger} \hat{f}_{\circ}^{\circ}\right\rangle$ for any operator $\hat{f}$.

We reformulate the condition (6) solely in terms of field correlation functions. Inserting Eq. (2) into the lhs of Eq. (6), we obtain a quadratic form:

$$
\left\langle\circ \hat{f}^{\dagger} \hat{f}_{\circ}^{\circ}\right\rangle=\sum_{\left\{p_{i}, q_{i}, n_{i}, m_{i}\right\}=0}^{\infty} c_{\left\{p_{i}, q_{i}\right\}}^{*} c_{\left\{n_{i}, m_{i}\right\}}\left\langle\stackrel{\circ}{\circ}\left[\hat{E}^{(-)}(1)\right]^{n_{1}+q_{1}} \ldots\left[\hat{E}^{(-)}(k)\right]^{n_{k}+q_{k}}\left[\hat{E}^{(+)}(k)\right]^{m_{k}+p_{k}} \ldots\left[\hat{E}^{(+)}(1)\right]^{m_{1}+p_{1} \circ} \circ .\right.
$$

The necessary and sufficient condition for any violation of the nonnegativity of the quadratic form requires the negativity of at least one of the principal minors of the form (7). In view of the lengthy expressions we may only outline the procedure and consider some examples.

The nonclassicality conditions obtained from negativities of the second-order minor read as

$$
\begin{aligned}
& \left|\left\langle\circ\left[\hat{E}^{(-)}(1)\right]^{n_{1}+q_{1}} \ldots\left[\hat{E}^{(-)}(k)\right]^{n_{k}+q_{k}}\left[\hat{E}^{(+)}(k)\right]^{m_{k}+p_{k}} \ldots\left[\hat{E}^{(+)}(1)\right]^{m_{1}+p_{1}} \circ\right\rangle\right|^{2}>
\end{aligned}
$$

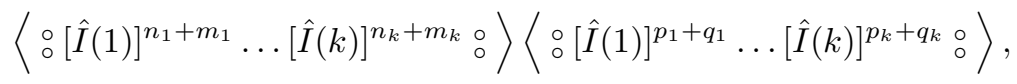

where $\hat{I}(i)=\hat{E}^{(-)}(i) \hat{E}^{(+)}(i)$ is the intensity operator. These conditions are sufficient for the existence of nonclassical correlations in the chosen space-time points. The necessary and sufficient conditions require the consideration of the minors of all higher orders.

Let us give an example for nonclassicality conditions based on third-order minors, leading to inequalities composed of sums of products of three correlation functions.
Specifying the minors is equivalent to start with a simplified form of the operator $\hat{f}$, such as

$$
\hat{f}=c_{1}\left[\hat{E}^{(+)}(1)\right]^{m}+c_{2}\left[\hat{E}^{(-)}(2)\right]^{n}+c_{3}:[\hat{I}(3)]^{p}:
$$

with redefined coefficients $c_{i}$. The resulting nonclassicality condition reads as

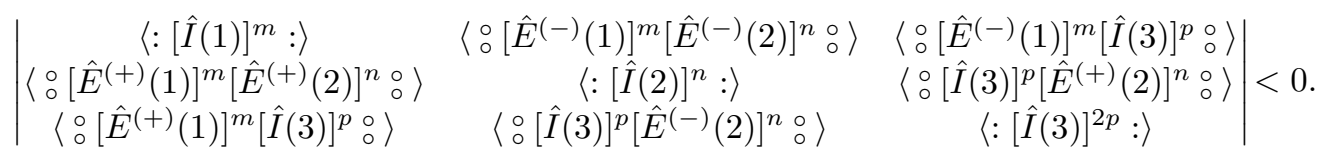

More generally, the minor contains more complex correlation functions, such as those in the condition (8).

We may further simplify the conditions (8). By choosing the only nonvanishing powers as $n_{1}=m_{1}=p_{2}=$ $q_{2}=1$, we get

$$
\langle\circ \hat{I}(1) \hat{I}(2) \circ\rangle>\sqrt{\left\langle:[\hat{I}(1)]^{2}:\right\rangle\left\langle:[\hat{I}(2)]^{2}:\right\rangle} .
$$

This is the photon-antibunching condition in its general form, which can be also derived as a violation of the
Schwarz inequality, for example cf. 22]. The intensity correlation function on the lhs is nonnegative, so that the absolute value can be omitted. In the given form the antibunching condition also applies for nonstationary radiation [23]. The stationary condition was used in the pioneering photon-antibunching experiment in resonance fluorescence [4].

It is straightforward to formulate higher-order generalizations of the antibunching condition (11). In Eq. (8) we choose for the nonvanishing powers $n_{1}=m_{1}=N-n$, 
$n_{2}=m_{2}=M-m, q_{1}=p_{1}=n$, and $q_{2}=p_{2}=m \quad(N \geq n$ and $M \geq m)$, which leads to

$$
\left\langle\circ[\hat{I}(1)]^{N}[\hat{I}(2)]^{M} \circ\right\rangle>\sqrt{\left\langle\circ[\hat{I}(1)]^{2(N-n)}[\hat{I}(2)]^{2(M-m)} \circ\right\rangle\left\langle\circ[\hat{I}(1)]^{2 n}[\hat{I}(2)]^{2 m} \circ\right\rangle} .
$$

If we further specify $m=M$ and $n=0$, the condition

$$
\left\langle\circ[\hat{I}(1)]^{N}[\hat{I}(2)]^{M} \circ\right\rangle>\sqrt{\left\langle:[\hat{I}(1)]^{2 N}:\right\rangle\left\langle:[\hat{I}(2)]^{2 M}:\right\rangle}
$$

represents a direct higher-order generalization of the antibunching condition (11).

From the nonclassicality condition (8) one may also obtain conditions for the normally and time-ordered correlations of intensity and field strength. Such correlations have been studied in the resonance fluorescence of a single atom 24 and their measurement has been analyzed [25, 26]. A recently proposed method of balanced homodyne correlation measurements combines the advantages of balanced homodyning with those of correlation techniques [27]. By using a strong local oscillator it allows one to detect correlation functions of higher orders, even when the overall quantum efficiency is small.

Let us consider a typical example. Choosing in Eq. (8) the non-zero powers as $n_{i}=m_{i}+p_{i}$ with $p_{i} \geq 0$, we obtain the condition

$$
\begin{gathered}
\left|\left\langle\circ\left[\hat{E}^{(-)}(1)\right]^{p_{1}} \ldots\left[\hat{E}^{(-)}(k)\right]^{p_{k}}[\hat{I}(k)]^{m_{k}} \ldots[\hat{I}(1)]^{m_{1}} \circ\right\rangle\right\rangle \mid \\
>\sqrt{\left.\left\langle\circ[\hat{I}(1)]^{2 m_{1}+p_{1}} \ldots[\hat{I}(k)]^{2 m_{k}+p_{k}} \circ\right\rangle\right)}
\end{gathered}
$$

It gives some insight in the nonclassical correlation properties of intensity and field strength operators, by comparing normally and time-ordered correlation functions containing the field strength (here the negative frequency part) with intensity correlation functions. In the lowest order we may choose $p_{1}=m_{2}=1$ as the only nonvanishing powers, which yields

$$
\left|\left\langle\circ \hat{E}^{(-)}(1) \hat{I}(2) \circ\right\rangle\right|>\sqrt{\left\langle\circ \hat{I}(1)[\hat{I}(2)]^{2} \circ\right.} \circ \text {. }
$$

We can also formulate conditions for intensity-field strength correlations of other types. Let us restrict the generality of the operator $\hat{f}$, for example by setting

$$
\hat{f}=c_{1} \hat{E}^{(+)}(1)+c_{2} \hat{I}(2)
$$

Inserting this expression into the condition (6), we get

$$
\left|\left\langle\circ \hat{E}^{(-)}(1) \hat{I}(2) \circ\right\rangle\right|>\sqrt{\langle\hat{I}(1)\rangle\left\langle:[\hat{I}(2)]^{2}:\right\rangle} .
$$

In general it depends on the chosen radiation sources, in particular on their intensity correlation properties, whether this form of the condition is stronger than the form (15) or vice versa.

It is also of interest to formulate nonclassicality conditions including the full field strength. Equating the coefficients of $\hat{E}^{(-)}(1)$ and $\hat{E}^{(+)}(1)$ in the operator $\hat{f}$, the positive frequency part in Eq. (16) is replaced with the field strength operator, $\hat{E}(1)=\hat{E}^{(-)}(1)+\hat{E}^{(+)}(1)$. Now the nonclassicality condition is of the form

$$
|\langle\circ \hat{\circ}(1) \hat{I}(2) \circ\rangle|>\sqrt{\left\langle:[\hat{E}(1)]^{2}:\right\rangle\left\langle:[\hat{I}(2)]^{2}:\right\rangle} .
$$

The field strength-intensity correlations are no longer compared with the quantum statistical properties of the intensity alone. Note that nonclassical correlations of intensity and field strength of lowest order have been discussed under special conditions, such as for Gaussian fluctuations [26].

In the following we deal with the question of whether or not nonclassical correlation properties of the types introduced above may occur in the irradiation of realistic sources. As a simple example we consider the resonance fluorescence of a single two-level atom, for the theory cf. e.g. 22]. In this case we have detailed knowledge of the high-order correlation functions to be considered. In particular, the occurrence of photon antibunching is well known. The general concept of nonclassical correlations, however, applies to arbitrary radiation sources. For example, it could serve for a more complete characterization of the radiation in the mentioned cavity experiment [26], but also for many other radiation sources.

Let us start to consider nonclassical effects based on intensity correlation measurements of higher order, see the condition (12). When at least one of the values of $N$ or $M$ becomes two or larger, both sides of the inequality become zero, due to the fact that a single atom cannot simultaneously emit two photons. Higher-order nonclassical effects of this type do not occur in resonance fluorescence from a two-level atom, their observation requires other types of radiation sources.

Consider now the situation for field strength-intensity correlations. Whenever a term $\left[\hat{E}^{( \pm)}(i)\right]^{n}$ with $n \geq 2$ occurs $(i=1, \ldots, k)$ on the lhs of the condition (8), the corresponding correlation function vanishes for the single-atom resonance fluorescence. That is, such types 
of nonclassical correlations do not exist. Based on this knowledge, however, we may formulate a variety of nonclassicality conditions in terms of those higher-order correlation functions which do not contain terms of the mentioned type. This leads to inequalities of the form

$$
\begin{aligned}
& \left|\left\langle\circ \hat{E}^{(-)}(1) \ldots \hat{E}^{(-)}(l) \hat{I}(l+1) \ldots \hat{I}(k) \circ\right\rangle\right|> \\
& \left.\sqrt{\left\langle\begin{array}{c}
\circ \\
\circ
\end{array}(1) \ldots \hat{I}(l)[\hat{I}(l+1)]^{2} \ldots[\hat{I}(k)]^{2} \circ\right.} \circ\right\rangle,
\end{aligned}
$$

for $1<l<k$. To provide a nontrivial characterization of nonclassical correlations in single-atom resonance fluorescence, the retarded times $t_{i}-r_{i} / c$ must not become equal for two space-time points. It is easy to verify by resonance fluorescence theory that such nonclassical correlations exist for a two-level atom, since the rhs of the condition (19) becomes zero. For getting more insight in the full time-dependent correlation properties of higher orders on the lhs, the correlation functions of the resonance fluorescence can be calculated by standard methods, such as the quantum regression theorem, see e.g. [22]. An experimental demonstration clearly requires the detection of the correlation functions occuring in the condition (19). Note that one may consider a manyfold of other conditions for nonclassical space-time dependent correlations by using higher-order minors of the general quadratic form (7), for example cf. inequality (10).

An open problem is the application of space-time dependent nonclassicality conditions for characterizing entanglement of multimode radiation fields. Since the $P$ functions of entangled states fail to be probability densities, they are included in the conditions derived above. To distinguish entanglement from other nonclassical effects requires to handle time-dependent field commutation rules, which is a nontrivial task that requires further research. For details on normal- and time-ordering and time-dependent commutation rules of source-attributed quantized radiation we refer to [22].

In summary, we have studied the physical properties of nonclassical space-time dependent correlations: their complete characterization, the realization of such effects in resonance flourescence, and their observation by balanced homodyne correlation measurements. In general nonclassicality is characterized by negativities of properly arranged minors of various orders, whose entries are normally and time-ordered field correlation functions of arbitrarily high orders. The conditions completely describe the nonclassical correlation properties, including entanglement, of any realistic radiation field whose generalized Glauber-Sudarshan $P$-functional fails to be a joint probability density. This concept applies not only to mul- timode quantum states in a nonclassical initial preparation, but also to nonclassical properties emerging from the dynamics of radiation sources.

The author gratefully acknowledges valuable comments by C. Di Fidio, M. Fleischhauer, Th. Richter and E. Shchukin. This work was supported by the Deutsche Forschungsgemeinschaft.

* Electronic address: werner.vogel@uni-rostock.de

[1] A. Einstein Ann. Phys. 17, 132 (1905).

[2] E. Schrödinger, Naturwiss. 23, 807 (1935).

[3] A. Einstein, B. Podolsky, and N. Rosen, Phys. Rev. 47, 777 (1935).

[4] H. J. Kimble, M. Dagenais, and L. Mandel, Phys. Rev. Lett. 39, 691 (1977).

[5] R. Short and L. Mandel, Phys. Rev. Lett. 51, 384 (1983).

[6] R. E. Slusher, L. W. Hollberg, B. Yurke, J. C. Mertz, and J. F. Valley, Phys. Rev. Lett. 55, 2409 (1985).

[7] E. Sudarshan, Phys. Rev. Lett. 10, 277 (1963).

[8] R. J. Glauber, Phys. Rev. 131, 2766 (1963).

[9] U. Titulaer and R. Glauber, Phys. Rev. 140, B676 (1965).

[10] L. Mandel, Phys. Scr. T12, 34 (1986).

[11] W. Vogel, Phys. Rev. Lett. 84, 1849 (2000).

[12] T. Richter and W. Vogel, Phys. Rev. Lett. 89, 283601 (2002).

[13] E. Shchukin, T. Richter, and W. Vogel, Phys. Rev. A 71, 011802(R) (2005).

[14] E. V. Shchukin and W. Vogel, Phys. Rev. A 72, 043808 (2005).

[15] A. I. Lvovsky and J. H. Shapiro, Phys. Rev. A 65, 033830 (2002).

[16] A. Zavatta, V. Parigi, and M. Bellini, Phys. Rev. A 75, 052106 (2007).

[17] E. Shchukin and W. Vogel, Phys. Rev. Lett. 95, 230502 (2005).

[18] G. S. Agarwal and A. Biswas, New Journal of Physics 7, 211 (2005).

[19] M. Hillery and M. S. Zubairy, Phys. Rev. Lett. 96, 050503 (2006).

[20] E. Shchukin and W. Vogel, Phys. Rev. A 74, 030302(R) (2006).

[21] A. Miranowicz, M. Piani, P. Horodecki, and R. Horodecki, quant-ph/0605001.

[22] W. Vogel and D.-G. Welsch, Quantum Optics (WileyVCH, 2006), 3rd ed.

[23] A. Miranowicz, J. Bajer, H. Matsueda, M. Wahiddin, and R. Tanaś, J. Optics B 1, 511 (1999); 603 (1999).

[24] W. Vogel, Phys. Rev. Lett. 67, 2450 (1991).

[25] W. Vogel, Phys. Rev. A 51, 4160 (1995).

[26] H. J. Carmichael, H. M. Castro-Beltran, G. T. Foster, and L. A. Orozco, Phys. Rev. Lett. 85, 1855 (2000).

[27] E. Shchukin and W. Vogel, Phys. Rev. Lett. 96, 200403 (2006). 